\title{
'Engagement' y redes sociales. Análisis bibliométrico desde el ámbito científico de las relaciones públicas
}

\section{Engagement and Social Media. Bibliometric Analysis from the Scientific Field of Public Relations}

Ángeles Moreno-Fernández

Universidad Rey Juan Carlos (España)

María Cristina Fuentes-Lara

Universidad Rey Juan Carlos / Universidad Francisco de Vitoria (España)

Uno de los aspectos de mayor influencia de las redes sociales es el engagement de los stakeholders, mediante el cual una empresa puede conversar con sus grupos de interés. El objetivo del presente trabajo es realizar un análisis bibliométrico que permita identificar la importancia y la evolución del engagement en redes sociales en el ámbito de las empresas dentro de la literatura especializada en relaciones públicas y gestión de comunicación.

Un análisis de contenido cuantitativo bibliométrico nos ha permitido evaluar la actividad y la producción científica de las publicaciones que aparecen en las revistas académicas con mejor posición en JCR y Scopus en el ámbito de gestión de las relaciones públicas en el período comprendido entre 2010 y 2017. Para el análisis se han incluido indicadores de producción, dispersión, colaboración, literatura científica, rigurosidad empírica y asociaciones temáticas.
One of the most influential aspects of social media is the engagement with stakeholders, thanks to which a company or organization can converse with its interest groups. The objective of this work is to carry out a bibliometric analysis to identify the importance and evolution of engagement on social media in the area of companies and organizations by reviewing the specialized literature about Public Relations and Communication Management.

A quantitative bibliometric content analysis has enabled us to assess the scientific activity and production of papers published in academic journals in top JCR and Scopus positions in the field of Public Relations between 2010 and 2017. Diverse indicators have been included to pinpoint production, dissemination, collaboration, scientific literature, scientific accuracy and thematic patterns.

Regarding the indicators for dissemination, results show a gradual increase 
Los resultados evidencian un crecimiento gradual durante los años de estudio de la productividad científica sobre la temática objeto de este estudio, de acuerdo con los indicadores de dispersión. Se han detectado diez categorías temáticas principales y algunas tendencias en cuanto a la autoría y el uso de referencias.

Tras analizar todos los datos queda patente la importancia que adquiere el engagement en redes sociales en el ámbito de las relaciones públicas. Es especialmente notable el número de artículos referentes al análisis de la responsabilidad social corporativa (RSC), la comunicación de crisis, la legitimidad o la reputación, debido al creciente interés que tienen las empresas en mejorar y profundizar en la relación que mantienen con sus públicos.

Palabras clave: engagement, relaciones públicas, gestión de comunicación, social media, bibliometría. of scientific productivity on the topic of study during the period under scrutiny. Ten thematic patterns have been identified along with common tendencies regarding authorship and references.

After analysing the data, it is evident the importance that engagement on social media has acquired for the field of Public Relations. It is especially noteworthy the number of papers linked to corporate social responsibility, crisis communication, legitimacy and reputation, on account of the interest of organizations in improving and strengthening relations with their target.

Key words: engagement, public relations, communication management, social media, bibliometrics.

【 a satisfacción de las necesidades de los clientes se ha convertido en un aspecto fundamental para el desarrollo de ventajas competitivas (Mpinganjira, 2016). Los usuarios de la sociedad postmoderna buscan la satisfacción de necesidades compuestas, incluida la necesidad de desempeñar roles más activos en el proceso de consumo y comunicación (Di Gangi y Wasko, 2016; Gambetti y Graffigna, 2010; Shahbannejad, Dolan y Tripathi, 2017;). El 40,50\% de la población utiliza a diario las redes sociales (ONTSI, 2017), de los cuales el 16\% las usan entre otras cosas para hablar en la red de productos y servicios, y llegan ya a un 83\% los que siguen a sus marcas (IAB, 2017: 30-42).

Por estas razones, diversos autores subrayan que la presencia de las organizaciones y empresas en redes sociales y el uso de estrategias correctas es imprescindible hoy y ha transformado la forma de gestionar los negocios (Kietzmann, Hermkens y Mccarthy, 2011; Tiago y Verrísimo, 2014).

Las organizaciones están teniendo en cuenta cada vez más el efecto de los comportamientos no transaccionales en sus resultados (Kumar et al., 2010). Las 
redes sociales les brindan la oportunidad de uso de una variedad de herramientas para personalizar la comunicación y generar la implicación emocional con sus diversos stakeholders.

Uno de los campos de la comunicación organizacional en el que la presencia de las redes sociales influirá más notablemente es el engagement de los stakeholders, con las posibilidades de generar conversaciones con ellos y conocer de primera mano sus respuestas para generar una mayor legitimidad (Sixto, Aguado y Rivero, 2017).

\section{MARCO DE LA INVESTIGACIÓN}

A pesar de la falta de claridad sobre el concepto, la investigación sobre el engagement ha estado en auge, impulsada por la creciente popularidad de las redes sociales y la lucha de las organizaciones para involucrar a los públicos digitalmente.

Dhanesh (2017) señala que la mayor parte del trabajo académico sobre el engagement ha tenido lugar en áreas de la psicología aplicada, la educación (engagement académico), el marketing y la publicidad (compromiso del cliente), el comportamiento organizacional y la gestión de recursos humanos (engagement de los empleados y burnout), y las ciencias políticas (compromiso cívico y político).

Desde el ámbito de la comunicación con consumidores y clientes, Brodie et al. (2011) indican que el engagement del consumidor es un estado psicológico que se produce en virtud de la interactividad y experiencias compartidas de los clientes con las marcas y que depende del nivel de intensidad con el que ocurren dichas interacciones y del contexto en el que se sitúen.

Van Doorn et al. (2010) y Muñoz, Oviedo y Castellanos (2017) defienden que la participación del cliente va más allá del simple acto de compra, ya que los clientes, por medio del engagement, se involucran profundamente con las marcas. Como ejemplo de esto, podemos considerar el hecho de que los clientes que están comprometidos y tienen una relación con la marca tienen más probabilidades de realizar comentarios positivos sobre su experiencia con la marca a familiares y amigos, lo que genera todo un proceso de boca a boca beneficioso para la marca. En consecuencia, podrían convertirse en defensores de la marca y generar valor para ella. Sin embargo, cabe señalar que la participación del cliente también puede tener un impacto negativo en las marcas. Considerando un ejemplo análogo al anterior, si los clientes comienzan a compartir eWOM ("boca a boca" electrónico) negativo, las marcas sufrirán publicidad negativa y los clientes potenciales podrían decidir no invertir en ella. Por lo tanto, es trascendental para las marcas gestionar adecuadamente el engagement del cliente (Hollebeek, Leventhal y Chen, 2014). Estas aproximaciones dejan claro que los comportamientos del cliente comprometido exceden la mera transacción y el consumo (Zhang et al., 2017).

Gambetti y Grafigna (2010) señalan que los ámbitos más interesantes para empresas e investigadores son el engagement cliente/consumidor y el engagement de la marca, "lo que proporciona una orientación hacia un concepto holístico e integrado del engagement, que no está ligado a un medio o mensaje en particular, sino a una estrategia de marca global dirigida a un mercado específico". 
Si ampliamos la visión a otros stakeholders, en el ámbito holístico de las relaciones públicas y la gestión de la comunicación, los tres bloques principales que debemos tener en cuenta son el engagement digital, el engagement de los empleados y el engagement cliente/consumidor (Dhanesh, 2017). En esta misma perspectiva, Tylor y Kent (2014) sitúan el engagement dentro del concepto de comunicación y diálogo éticos, centrándose en la dimensión del diálogo de proximidad. Es más, previo a la expansión del concepto ligado a las redes sociales, Como plantea Pieczka (2014) sitúa el principio del engagement dialógico como base ética para las relaciones públicas ha proporcionado uno de los ejes para el desarrollo de la teoría de relaciones públicas desde los años ochenta.

Dhanesh (2017) define al engagement como un estado afectivo, cognitivo y conductual en el que el público y las organizaciones que comparten intereses mutuos interactúan colaborando de forma activa y pasiva para lograr la consecución de objetivos comunes.

Siguiendo estas premisas, observamos cómo la complejidad de este concepto no permite su definición mediante un único significado, sino que se requiere de la combinación de diferentes ideas vinculadas para poder entenderlo y los diversos enfoques desde distintas ramas de la comunicación se van influenciando mutuamente en el conocimiento del fenómeno. La mayoría de autores comparten esta aproximación multidimensional del engagement, ya sea bidimensional (ejemplo Mollen y Wilson, 2010); tridimensional (ejemplo Brodie et al., 2011; Dessart, Veloutsou y Morgan-Thomas, 2015; Dhanesh, 2017; Hollebeek et al., 2016); cuatridimensional (ejemplo Islam y Rahman, 2016; Vivek et al., 2014) o quintupledimensional (ejemplo Gambetti y Grafigna, 2010; Kanm et al., 2016; Van Door et al., 2010).

La siguiente tabla recoge las definiciones más relevantes de engagement desde el punto de vista de comunicación de marca y de relaciones públicas:

\section{Tabla 1. Definiciones de 'engagement'}

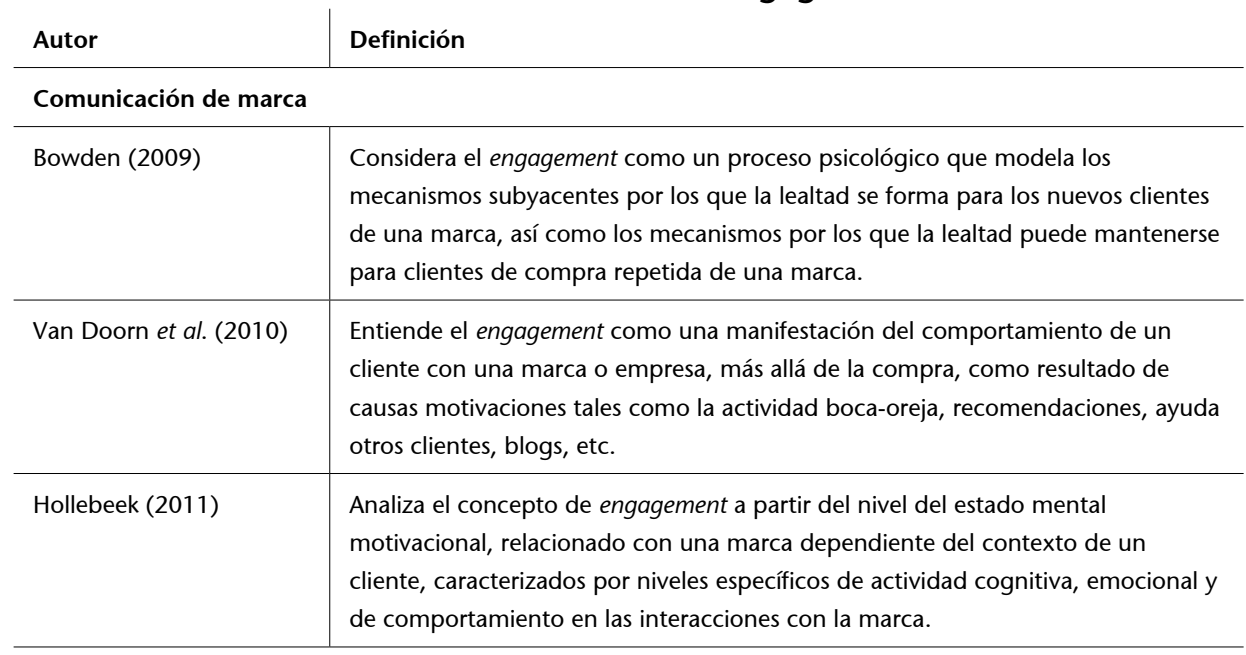




\begin{tabular}{l|l} 
Autor & Definición \\
\hline Morgan et al. (2012) & $\begin{array}{l}\text { Entiende el engagement como la intensidad de la participación del consumidor y } \\
\text { su conexión con las ofertas de la organización y / o sus actividades }\end{array}$ \\
\hline Hollebeek et al. (2014) & $\begin{array}{l}\text { Considera el engagement como algo positivo para el consumidor al considerarlo } \\
\text { como una actividad cognitiva, emocional y de conducta relacionada con la } \\
\text { marca que se produce durante las interacciones específicas consumidor / marca. }\end{array}$ \\
\hline Relaciones públicas y gestión de la comunicación \\
\hline $\begin{array}{l}\text { Gambetti y Graffigna } \\
\text { (2010) }\end{array}$ & $\begin{array}{l}\text { Estudia el engagement como un concepto complejo, fuertemente influido por } \\
\text { componentes psicológicos, sociales, interactivos, relacionales, experienciales y } \\
\text { basados en el contexto. Es un fenómeno postmoderno, con múltiples facetas y } \\
\text { difícil de predecir pues implica interacción entre individuos y entre estos y sus } \\
\text { contextos. }\end{array}$ \\
\hline Dhanesh (2017) & $\begin{array}{l}\text { Define el engagement como un estado afectivo, cognitivo y conductual en } \\
\text { el que los públicos y las organizaciones que comparten intereses mutuos en } \\
\text { temas destacados interactúan en grupos que oscilan entre pasivos y activos y } \\
\text { entre control y colaboración, y están dirigidos al logro de objetivos, ajustes y } \\
\text { adaptación para públicos y organizaciones. }\end{array}$ \\
\hline
\end{tabular}

Fuente: adaptada y completada a partir de Brodie et al. (2011) y Hollebeek (2011).

A raíz de las definiciones anteriores se puede ver la relevancia que tiene en la literatura académica y en el mundo de los negocios el concepto de engagement. No obstante, cabe señalar que la mayoría de los estudios se han realizado desde el campo de la comunicación de marketing y/o de marca, mientras que hay muy pocos estudios en el campo de las relaciones públicas y gestión de comunicación. Por todo ello, consideramos que es importante hacer un estudio bibliométrico con el fin de localizar y ordenar las aportaciones en este ámbito.

\section{METODOLOGÍA}

Se ha realizado un estudio bibliométrico de cinco de las principales revistas de comunicación entre los años 2010 y 2017 con el fin de caracterizar el inicio, desarrollo y el estado actual del engagement en las redes sociales y para estudiar y analizar la producción científica relativa a este campo.

Hemos elegido esta técnica de análisis de datos porque la bibliometría es uno de los métodos de estudio métrico más fiables, consistentes, plurales y reconocidos para determinar la productividad científica (Peña, 2012). La bibliometría utiliza en un principio métodos estadísticos y matemáticos para cuantificar las comunicaciones o las producciones científicas (Pritchard, 1969; Tague-Sutcliffe, 1994).

No obstante, el concepto se puede ampliar distinguiendo entre bibliometría descriptiva, que trata de aspectos puramente cualitativos y bibliometría evaluativa, que complementa a la primera con estudios de evaluación de la actividad científica mediante la aplicación de técnicas estadísticas y programas informáticos de mayor complejidad (Peña, 2012). 
La bibliometría como método científico se basa en la investigación de comportamientos estadísticamente regulares de los distintos factores relacionados con la producción científica. Se apoya en leyes como la de crecimiento exponencial, la de productividad de los autores o la de dispersión de la literatura científica (Castillo y Carretón, 2010). Para medir los resultados de la actividad científica necesitamos herramientas que nos ofrezcan información específica sobre cada campo, conocidas como indicadores científicos. En este estudio vamos a utilizar los siguientes indicadores:

- Indicadores de producción: son aquellos que ponen el foco de atención en medir factores relacionados con las publicaciones. Hacen recuento del número de páginas de cada artículo, el número de revistas o la cantidad de artículos que han escrito los distintos autores, universidades, instituciones o países (productividad científica). También se puede determinar el índice de producción, que es el número de autores que han participado en el 50\% de los artículos, y el índice de transitoriedad, que hace referencia a la cantidad de trabajos con un solo autor (Castillo y Carretón, 2010).

- Indicadores de dispersión: se centran en las revistas científicas, siendo útiles para determinar la cantidad de revistas especializadas por sector científico a partir de sus publicaciones. Se pueden medir también el número de artículos que tiene cada revista (Castillo y Carretón, 2010).

- Indicadores de colaboración: a partir del análisis de los autores que han participado en cada trabajo, estos indicadores estudian el grado de colaboración existente entre los distintos investigadores (Castillo y Carretón, 2010).

- Indicadores de uso de la literatura científica: se centran en el recuento y análisis de las referencias científicas que aparecen en los artículos publicados. Este tipo de datos nos pueden ayudar a determinar los trabajos más relevantes para un ámbito científico concreto, que serán aquellos que aparezcan en más artículos científicos (Castillo y Carretón, 2010).

- Indicadores de rigurosidad empírica: estudian para cada trabajo la variable metodológica utilizada para realizar la investigación (Castillo y Carretón, 2010).

- Indicadores de asociaciones temáticas: permiten analizar las referencias bibliográficas comunes para realizar una selección de artículos con temáticas similares, hacer un análisis de citas comunes para ver el grado de asociación entre varios documentos y, con el análisis de palabras comunes, estudiar la relación de palabras clave que describen el contenido del trabajo y que ayudan a indizar los trabajos científicos (Sancho, 1990).

\section{Selección de LA MUESTRA}

Con el fin de alcanzar los objetivos planteados, se ha aplicado un modelo de "búsqueda avanzada". Este modelo admite ajustar la búsqueda empleando varias palabras clave, lo que posibilita caracterizar el perfil de los artículos a escoger centrándonos en campos como: Título, Palabras Clave y Resumen. Además, se ha utilizado también un filtro por intervalo de tiempo para centrarnos en los trabajos realizados entre los años 2010 y 2017. 
Como síntesis de lo anterior, y con el fin de optimizar el proceso de búsqueda, desarrollamos la siguiente ecuación:

Tabla 2. Ecuación propuesta para realizar la búsqueda

( $\mathrm{TI}$ (engagement) OR IF(engagement) OR AB(engagement)) AND (TI("social media") OR IF(social media) OR $\mathrm{AB}$ (social media) OR $\mathrm{TI}$ (communication) OR IF(communication) OR AB(communication) OR TI(organization) OR IF(organization) OR AB(organization) OR TI(brand) OR IF(brand) OR AB(brand) OR TI(company) OR IF(company) OR AB(company) OR TI(relationship) OR IF(relationship) OR AB(relationship))

engagement AND ("Social Media" OR Communication OR Organization OR Brand OR Company OR Relationship)

* En caso de que la base de datos permita hacer la selección de los campos (TITLE-ABS-KEY) Fuente: elaboración propia.

Este modelo se ha aplicado a las siguientes bases de datos:

Tabla 3. Bases de datos utilizadas para la búsqueda

\begin{tabular}{l|l} 
Bases de datos & $\begin{array}{l}\text { No de resultados } \\
\text { encontrados }\end{array}$ \\
\hline ABI/INFORM Collection & 41.028 \\
\hline Academic Search Complete & 14.657 \\
\hline Business Source Complete & 13.771 \\
\hline Communication \& Mass Media Complete & 1.666 \\
\hline ProQuest Research Library & 14.897 \\
\hline ScienceDirect & 4.129 \\
\hline Scopus & 45.919 \\
\hline TOTAL & 136.067 \\
\hline
\end{tabular}

Fuente: elaboración propia.

Se ha obtenido un elevado número de resultados, ascendiendo a un total de 136.067 artículos. Este gran volumen de información es difícilmente procesable con los medios disponibles, por lo que se requiere acotar y centrar la búsqueda ciñéndonos únicamente a revistas de comunicación y relaciones públicas, que es la materia objeto del estudio del presente trabajo. Por lo tanto, hemos centrado la búsqueda en 5 revistas especializadas en comunicación y relaciones públicas con mejor posicionamiento en los índices JCR y Scopus en 2017, hemos obtenido un total de 642 resultados. Adicionalmente, se ha aplicado el filtro de la búsqueda avanzada a través de la ecuación mencionada anteriormente, con un resultado de un total de 141 artículos encontrados.

Finalmente se ha realizado la lectura y comprobación detallada de cada uno de los 141 artículos, analizando la temática y la metodología empírica de los mismos. Tras hacer este estudio se ha determinado que 44 artículos tratan sobre 
56 engagement en redes sociales en el ámbito de las empresas, que es el objeto de estudio de nuestro trabajo.

\begin{tabular}{|c|c|c|c|c|}
\hline Revista & $\begin{array}{l}\text { № de } \\
\text { publicaciones } \\
\text { con la } \\
\text { palabra clave } \\
\text { 'engagement' }\end{array}$ & $\begin{array}{l}\text { № de } \\
\text { publicaciones } \\
\text { utilizando la } \\
\text { ecuación }\end{array}$ & $\begin{array}{l}\text { № de } \\
\text { publicaciones } \\
\text { seleccionados } \\
\text { para el estudio }\end{array}$ & Base de datos \\
\hline Public Relations Review & 325 & 75 & 22 & ScienceDirect \\
\hline $\begin{array}{l}\text { Journal of } \\
\text { Communication } \\
\text { Management }\end{array}$ & 91 & 19 & 7 & $\begin{array}{l}\text { ABI/INFORM Collection } \\
\text { Scopus } \\
\text { Google Academic }\end{array}$ \\
\hline $\begin{array}{l}\text { International } \\
\text { Journal of Strategic } \\
\text { Communication }\end{array}$ & 57 & 13 & 7 & $\begin{array}{l}\text { Communication \& Mass } \\
\text { Media Complete }\end{array}$ \\
\hline $\begin{array}{l}\text { Corporate } \\
\text { Communications: An } \\
\text { International Journal }\end{array}$ & 108 & 17 & 5 & ABI/INFORM Collection \\
\hline $\begin{array}{l}\text { Journal of Public } \\
\text { Relations Research }\end{array}$ & 61 & 17 & 3 & $\begin{array}{l}\text { Business Source } \\
\text { Complete } \\
\text { Communication \& Mass } \\
\text { Media Complete } \\
\text { LexisNexis Academic }\end{array}$ \\
\hline TOTAL & 642 & 141 & 44 & \\
\hline
\end{tabular}

Fuente: elaboración propia.

\section{Tratamiento DE DATOS}

Se ha extraído la información de los artículos seleccionados según los campos de la ficha técnica previamente diseñada para este estudio. Finalmente, se ha procesado la información recogida a través del análisis cruzado de contenidos. Este procesamiento se ha hecho mediante el uso de una hoja de cálculo de Microsoft Excel 2016 y UCINED 6.

\section{RESULTADOS}

\section{INDICADORES DE DISPERSIÓN}

Claramente podemos apreciar una tendencia al alza del número de artículos que tratan del engagement en redes sociales en el ámbito de las organizaciones publicados por año durante el periodo de estudio, incrementándose desde los 0 artículos editados en 2010 hasta los 17 publicados en 2017. 
Si ponemos el foco en la evolución del número de artículos por cada revista podemos destacar que Public Relations Review es la que tiene el mayor volumen de artículos publicados en el año 2017. Esto es debido a la sección especial sobre relaciones públicas y 'engagement' que publicó en diciembre de 2017.

La revista que mayor productividad ha tenido en el periodo estudiado es $\mathrm{Pu}$ blic Relations Review con 22 artículos, seguida de Journal of Communication Management e International Journal of Strategic Communication.

Gráfico 1. Productividad de artículos por revista y año

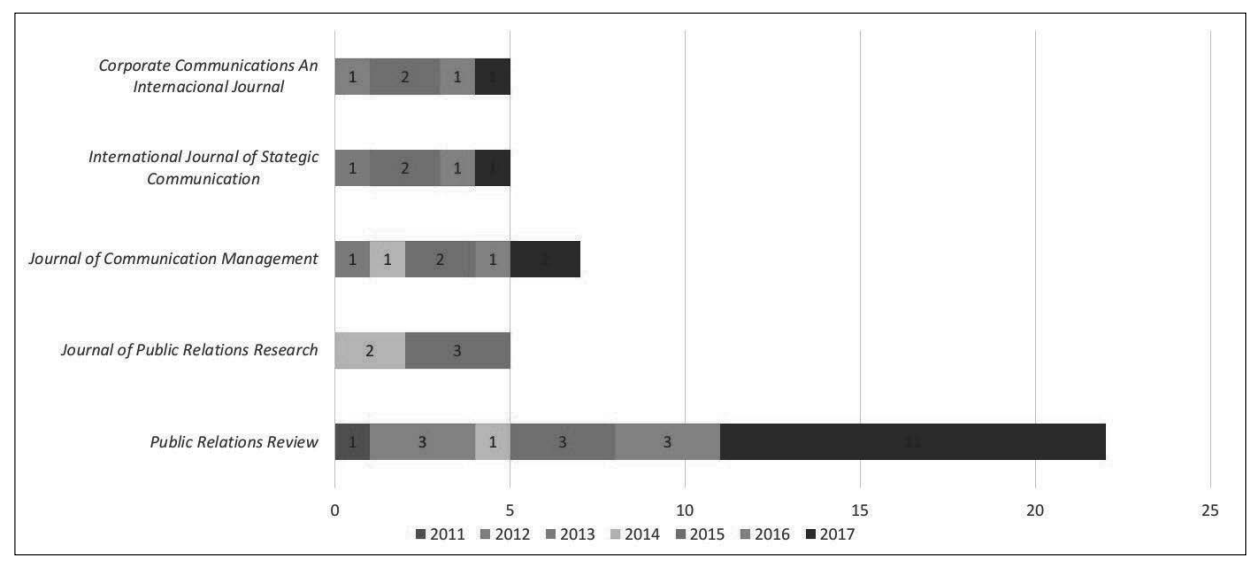

Fuente: elaboración propia.

\section{INDICADORES DE PRODUCCIÓN}

\section{Productividad por número de páginas}

Se observa que predominan los artículos que ocupan entre 7 y 13 páginas con el $52,27 \%$ de los valores analizados, que hacen un total de 23 artículos, seguidos de los de 14 a 19 páginas, que incumben a 13 artículos (29,55\%). El tercer grupo más numeroso lo forman los artículos que contienen entre 1 y 6 páginas, con un total de 7 publicaciones (el 15,91\% del total), y hay tan solo 1 artículo que ocupa más de 20 páginas (2,27\%). A continuación, se muestran los datos en una tabla para facilitar su visualización: 


\section{Gráfico 2. Productividad por número de páginas}

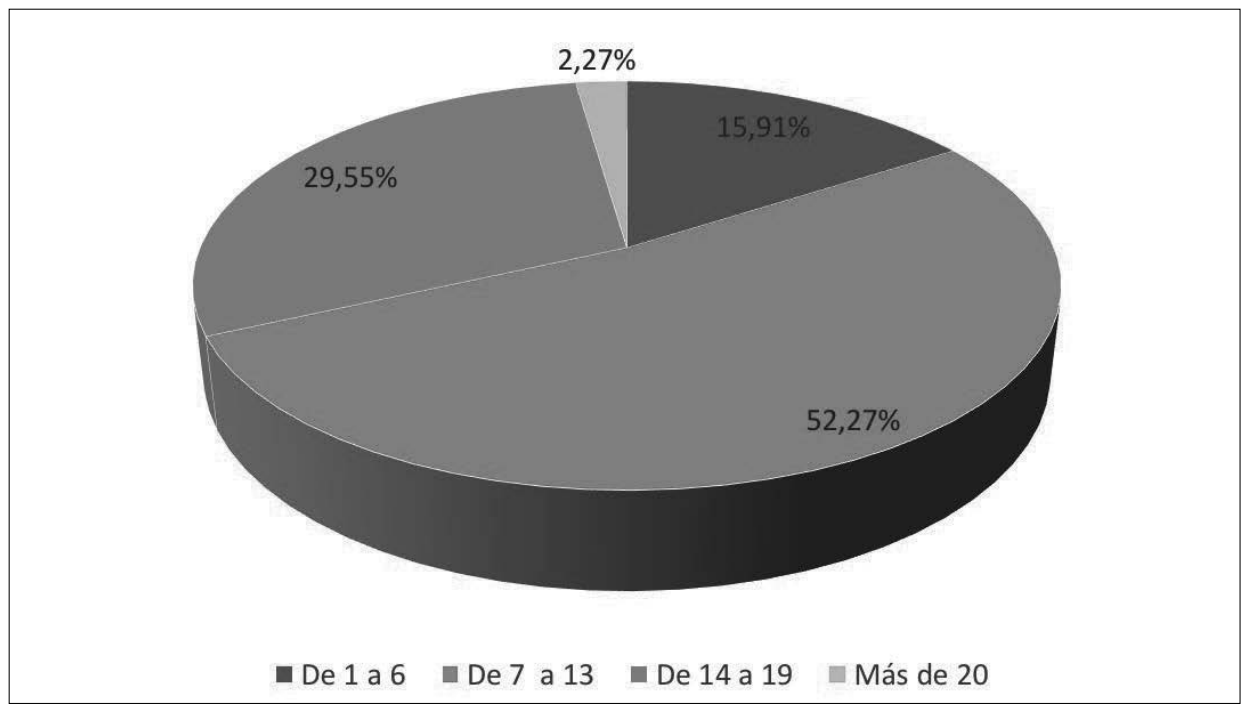

Fuente: elaboración propia.

\section{Productividad por países}

Para analizar este indicador bibliométrico de publicación por procedencia geográfica nos hemos basado en el origen de la institución a la que pertenece el primer autor firmante y no en el lugar de nacimiento de este, ya que queremos averiguar en qué países se están destinando más recursos al estudio del engagement en las redes sociales.

El estudio de los resultados nos ha permitido identificar en la muestra un total de 10 países, de entre los cuales destaca Estados Unidos como el país con más publicaciones, ya que ostenta un total de 33 artículos publicados que representan el $75 \%$ del total analizado. 
Gráfico 3. Productividad por países

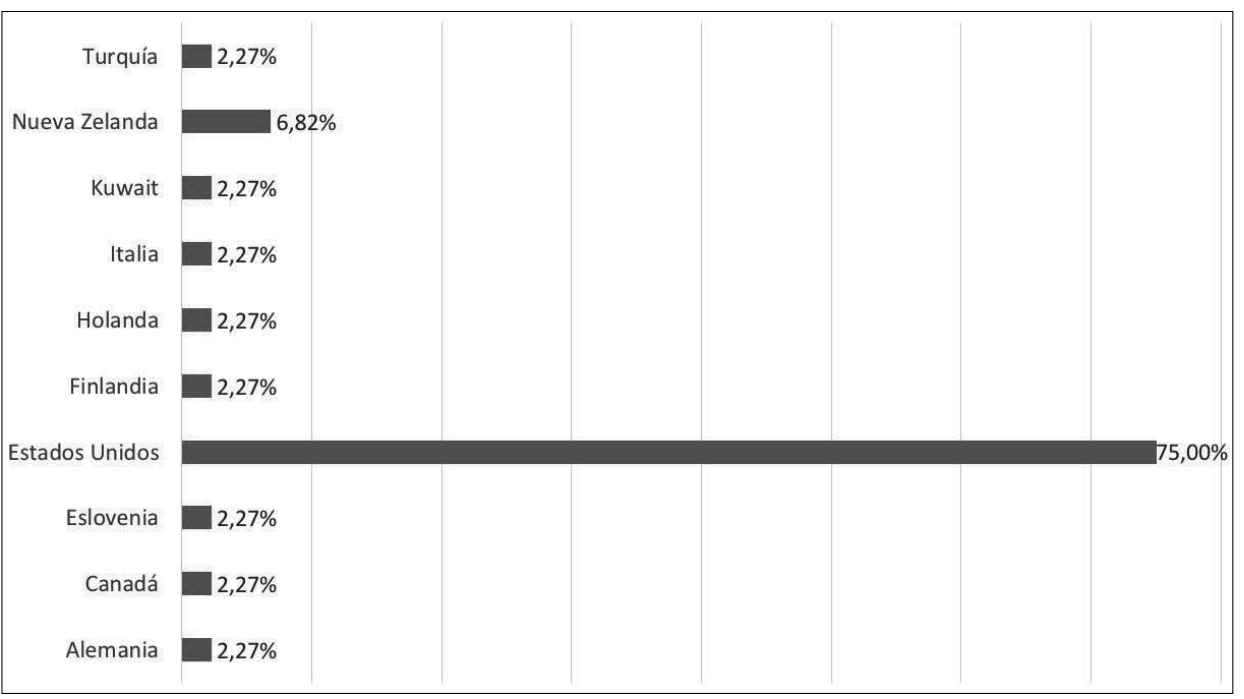

Fuente: elaboración propia.

\section{Productividad autoral}

Tras la recopilación de datos se analizaron todas las publicaciones para contabilizar el número de autores encontrados por artículo sin establecer ningún tope máximo ni agrupaciones. Se han identificado un total de 88 autores que han participado en los artículos seleccionados, y se han obtenido del estudio los siguientes resultados: el mayor porcentaje de productividad autoral lo recogen los artículos con dos autores, ya que de los 44 trabajos analizados 20 de ellos han sido producidos con la colaboración de dos personas $(45,45 \%)$. El segundo mayor grupo lo forman aquellas publicaciones en las que han participado tres autores, con el 31,82\% de los datos (14 artículos).

Estos datos coinciden en gran medida con los recogidos en el estudio realizado por Castillo y Carretón (2010). En dicho trabajo justifican que una gran parte de las publicaciones tienen más de un autor porque normalmente se desarrollan en proyectos de investigación llevados a cabo por varios miembros, pero no suelen superar los tres autores porque en el área de conocimiento de las ciencias sociales y jurídicas se penalizan en el ámbito curricular los artículos con un número superior de participantes. En nuestro caso, el 79,55\% de los artículos están escritos por dos o tres autores.

Si analizamos el cruce de datos entre el número de páginas y el número de autores, podemos observar que, tal y como se ha comentado anteriormente, el mayor número de artículos del estudio que nos ocupa son los que están firmados por dos autores, con 9 artículos de entre 7 y 13 páginas, con 8 artículos entre 14 y 19 páginas y con 3 artículos entre 1 y 6 páginas. 
Gráfico 4. Productividad por número de páginas y número de autores

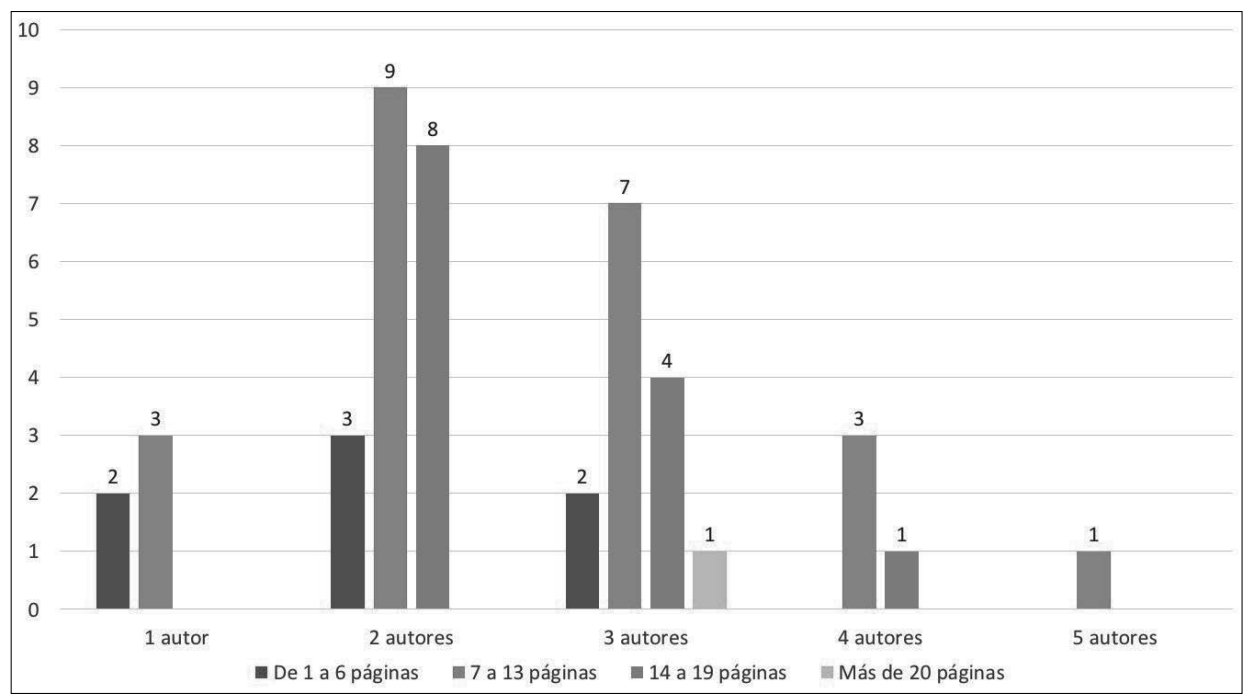

Fuente: elaboración propia.

\section{Procedencia de los autores o productividad institucional}

El trabajo de investigación referente a la procedencia de los autores ha sido llevado a cabo recopilando información sobre las universidades e instituciones a las que pertenecían todos los autores en el momento de realizar los artículos. El número de organismos diferentes suman un total de 61 , lo que refleja la elevada colaboración existente entre instituciones para realizar este tipo de estudios, ya que se han analizado un total de 44 artículos. Los tres organismos con mayor número de participantes son la Universidad de la Mancomunidad de Virginia $(7,34 \%$ de autores), la Universidad de Florida con un $6,42 \%$ de autores y la Universidad de Miami, con un 5,50\% de autores. Las tres más representativas son universidades de Estados Unidos. El anexo I recoge el total de instituciones que han participado en la elaboración de los artículos.

\section{INDICADORES DE COLABORACIÓN}

Para representar esta red de colaboración hemos utilizado un gráfico nodular, donde se puede apreciar que la comunidad de investigadores científicos que han participado en los artículos analizados se caracteriza, entre otras cosas, por trabajar preferiblemente de manera individual, puesto que la mayoría de los nodos son tan solo de 2 o 3 autores. No obstante, se puede observar que existen fuertes relaciones entre los autores más productivos, que están representados por los círculos más grandes. Entre ellos podemos destacar a Men y Tsai, con colaboraciones en 5 artículos cada uno. Otros autores como Wang y Waters colaboraron en 3 artículos, mientras que Guidry, Jin, Messner y Ji lo hicieron en 2. 
Gráfico 5. Red de colaboración autoral

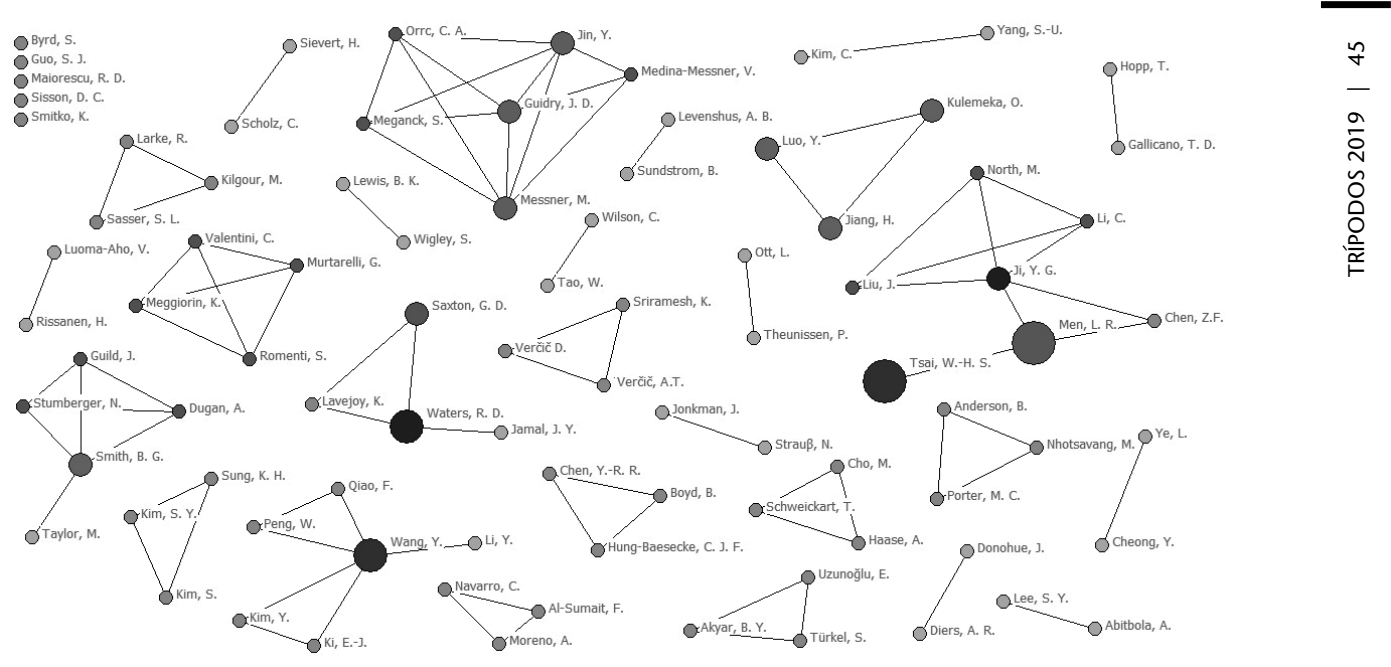

Fuente: elaboración propia.

Si relacionamos los países de procedencia de todos los autores participantes en los artículos, se aprecia que existe un trabajo colaborativo importante a escala internacional y se confirma que Estados Unidos forma parte del eje central de la investigación del engagement en redes sociales en el ámbito de las empresas a escala mundial. Como podemos observar en la siguiente gráfica, Estados Unidos colabora con instituciones y/o universidades de países como China, Nueva Zelanda, Croacia, Corea del Sur y Eslovenia. En muchos casos, estas colaboraciones son también una manifestación de una mayor concentración institucional, si tenemos en cuenta las universidades de doctorados y postgrados donde se han educado los autores.

\section{Gráfico 6. Red de colaboración por países y número de artículos}

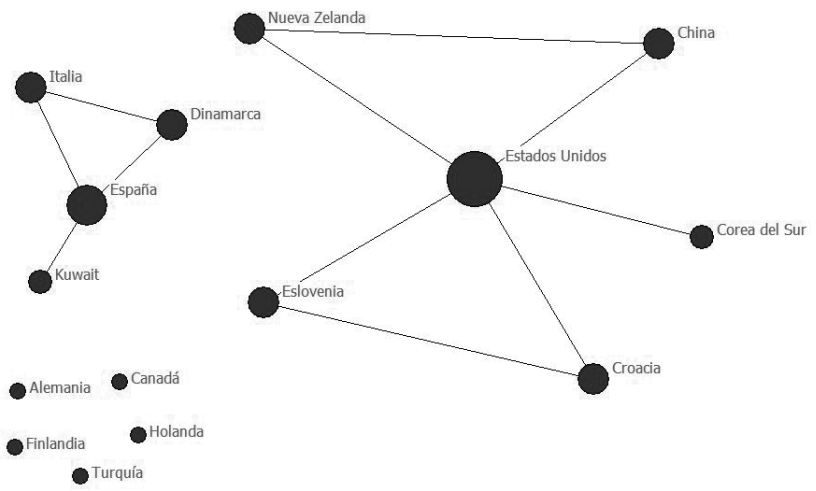

Fuente: elaboración propia. 
INDICADORES DE USO DE LA LITERATURA CIENTÍFICA

Se han contabilizado un total de 2.275 referencias bibliográficas en las 44 publicaciones analizadas, lo que corresponde a una media muy elevada de 51,7 referencias por cada trabajo. Desde el año 2012 se observa una escalada muy significativa en el número de referencias de media que aparecen en cada publicación.

Si analizamos el número de referencias en relación con el número de páginas por artículo se aprecia una relación entre la extensión de los artículos y las citas que recogen, determinando que a mayor número de páginas aumenta también el número de referencias. Esto es lógico, en la medida en que los autores deben acomodar sus trabajos de investigación a un número cada vez más limitado de páginas, lo que implica también reducir el número de referencias a las más sustanciales. A continuación, se muestra una gráfica en la que se aprecia la evolución del número de referencias por año y número de páginas:

\section{Gráfico 7. Relación entre el número de referencias y el número de páginas por años}

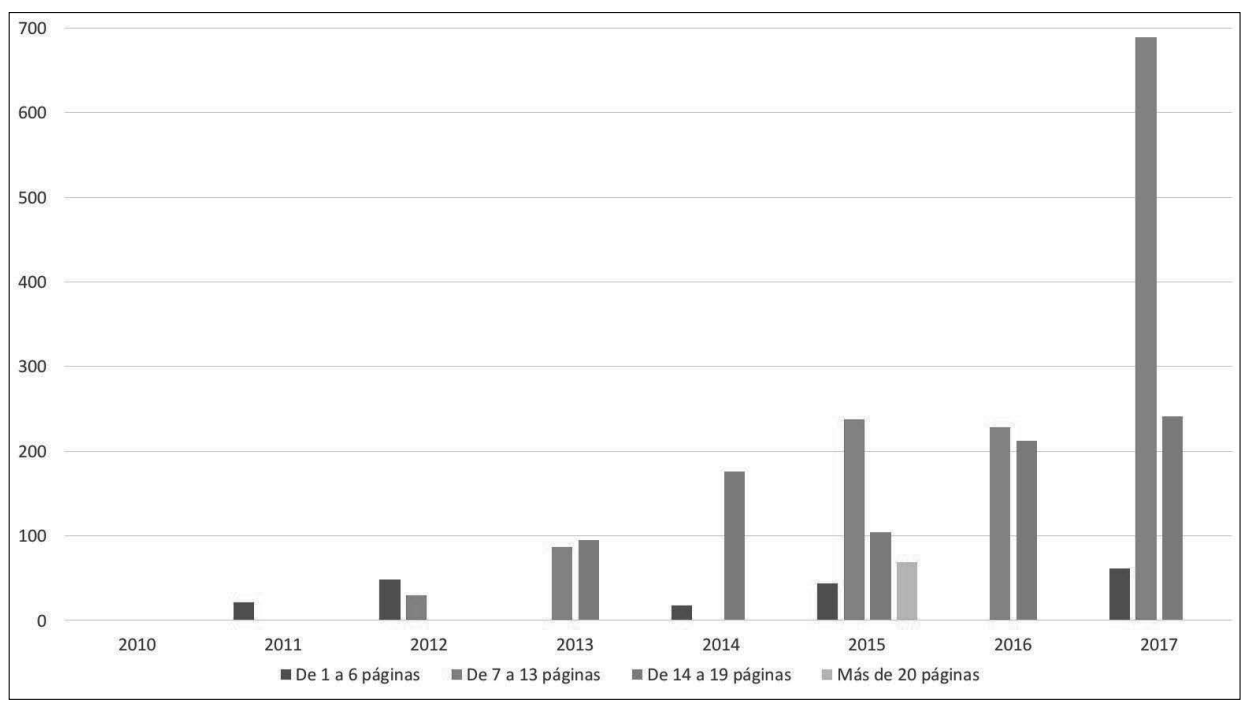

Fuente: elaboración propia.

Un apartado importante en el estudio de las referencias bibliográficas es el relativo a las autocitas. En este apartado hemos tenido en cuenta las citas referentes a artículos en los que cualquiera de los participantes aparece como autor principal en la bibliografía. De los 88 firmantes de los artículos analizados, tan solo 27 son los que se citaron a ellos mismos, representando el 30,68\%, frente a un $69,32 \%$ (61) que no lo han hecho (véase tabla 10). 
Tabla 5. Relación de autocitas de autores

\begin{tabular}{|c|c|c|}
\hline N.o & Autores que se han citado a sí mismos & Autocitas \\
\hline 1 & Men, L. R. & \\
\hline 2 & Tsai, W.-H. S. & \\
\hline 3 & Waters, R. D. & \\
\hline 4 & Smith, B. G. & \\
\hline
\end{tabular}

6

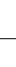

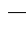

\begin{tabular}{|c|c|}
\hline 8 & Ji, Y. G. \\
\hline 9 & Kim, S. \\
\hline 10 & Saxton, G. D. \\
\hline 11 & Gallicano, T. D. \\
\hline 12 & Porter, M. C. \\
\hline 13 & Romenti, S. \\
\hline 14 & Sievert, $\mathrm{H}$. \\
\hline 15 & Wang, Y. \\
\hline 16 & Guidry, J. D. \\
\hline 17 & Hopp, T. \\
\hline 18 & Jiang, $\mathrm{H}$. \\
\hline 19 & Ki, E.-J. \\
\hline 20 & Maiorescu, R. D. \\
\hline 21 & Messner, M. \\
\hline 22 & Moreno, A. \\
\hline 23 & Sriramesh, K. \\
\hline 24 & Taylor, M. \\
\hline 25 & Theunissen, P. \\
\hline 26 & Verčič D. \\
\hline 27 & Yang, S.-U. \\
\hline
\end{tabular}

Fuente: elaboración propia.

Castillo y Carretón (2010) defienden que las autocitas de autor suelen darse en tres contextos: en los casos en que los autores tienen una elevada extensión de artículos, en los que los autores usualmente tienen un carácter autorreferenciador y en los que se dan ambas circunstancias. 
Además de las autocitas de autor debemos tener en cuenta las autocitas de revistas. Public Relations Review es la revista con más autocitas, con un promedio de 7 referencias. En segundo lugar está la revista Journal of Public Relations Research con un promedio de 6 citas.

\section{INDICADORES DE ASOCIACIONES TEMÁTICAS}

Dentro de las distintas categorías que se pueden analizar con este indicador nos vamos a centrar en el estudio de las referencias bibliográficas con temáticas comunes y en el análisis de las palabras clave que ayudan a indizar los trabajos científicos.

\section{Palabras clave}

En los 44 artículos analizados se ha encontrado un total de 218 palabras clave diferentes. Las 10 más utilizadas se refieren en la tabla 6.

Tabla 6. Productividad por palabras clave

\begin{tabular}{l|c} 
Palabras clave & No de repeticiones \\
\hline Social media & 30 \\
\hline Public relations & 8 \\
\hline Engagement & 7 \\
\hline Twitter & 7 \\
\hline Corporate communications & 5 \\
\hline Corporate Social Responsibility (CSR) & 4 \\
\hline Crisis communication & 4 \\
\hline Facebook & 4 \\
\hline Nonprofit organizations & 4 \\
\hline Strategic communication & 4 \\
\hline
\end{tabular}

Fuente: elaboración propia.

De aquí podemos extraer que las redes sociales Twitter y Facebook son las más analizadas en los estudios. Otro dato importante es que en el estudio del engagement, la responsabilidad social corporativa (RSC) y las organizaciones sin ánimo de lucro tienen una gran relevancia. Otros aspectos como la gestión de la comunicación en momentos de crisis empresariales son muy estudiados para comprobar si la relación de las marcas con sus consumidores es correcta en momentos críticos.

\section{Temática}

Se han determinado 10 categorías temáticas principales, de las cuales destacan la gestión de crisis y el engagement de los stakeholders en redes sociales, con 7 artículos cada una. La siguiente temática más numerosa es la de organizaciones sin 
ánimo de lucro, con 6 artículos. La responsabilidad social corporativa y el mensa-

je institucional en redes sociales les siguen con 5 artículos cada una.

Gráfico 8. Productividad por temática de los artículos

- Stakeholders engagement en redes sociales M Medición de engagement

- Líderes de opinión

- Empresas sin ánimo de lucro

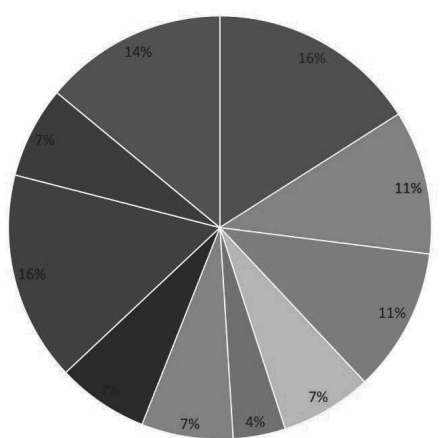

- Responsabilidad Social Corporativa - Marketing y engagement

- Gestión de crisis

- Mensaje institucional en redes sociales - Lideres empresariales

- Engagement de los empleados

Fuente: elaboración propia.

Podemos encontrar similitud entre los temas sobre los que tratan los artículos y las palabras clave más repetidas en los mismos, ya que las categorías más recurrentes y que parecen de mayor interés son las referentes al comportamiento de los consumidores en las redes sociales cuando se produce una crisis empresarial, debido a que una estrategia inapropiada puede destruir la reputación de la marca. La mejor forma de llegar a los stakeholders es a través de las plataformas digitales para establecer canales bidireccionales de comunicación, así como propiciar el boca a boca digital, cultivar relaciones a largo plazo, implementar el desarrollo de nuevos negocios y fomentar la creación de imagen y reputación de la empresa. Es también llamativo el número de estudios relativos a las organizaciones sin ánimo de lucro y la relación de estas con sus donantes.

\section{INDICADORES DE RIGUROSIDAD EMPÍRICA}

La herramienta de indicadores de rigurosidad empírica nos ayuda a determinar la variable metodológica que se ha utilizado en cada publicación para realizar la investigación, que puede ser de carácter teórico si en el artículo se cuestionan o se plantean determinados conceptos, enfoques o procedimientos, o de carácter empírico si se llegan a conclusiones a partir de la observación y análisis de diversos datos. En el caso de que la publicación esté fundamentada en un trabajo empírico también es relevante indicar las técnicas de investigación utilizadas para tal fin.

Tras analizar la metodología empleada en los 44 artículos seleccionados, todos los trabajos han sido de carácter empírico y no se ha recogido ningún estudio de carácter teórico. Dentro de este estudio, las técnicas más utilizadas han sido las 
cuantitativas, que se han repetido en 28 artículos. Les siguen, con 12 artículos, las técnicas cualitativas, y por último las investigaciones empíricas, que han empleado técnicas cuantitativas y cualitativas, presentes en 4 artículos. En la siguiente tabla se recogen las técnicas más relevantes utilizadas en cada caso, indicando el número de artículos en las que aparecen:

Tabla 7. Metodología empleada

\begin{tabular}{|c|c|}
\hline Metodología empleada & Total \\
\hline Empírica, con técnicas cualitativas & 12 \\
\hline Análisis contextual & 1 \\
\hline Análisis de contenido cualitativo & 2 \\
\hline Análisis del discurso & 1 \\
\hline Entrevista & 3 \\
\hline Entrevista en profundidad & 2 \\
\hline Entrevista en profundidad y cuestionario & 1 \\
\hline Grupos de discusión & 1 \\
\hline Estudio de caso múltiple & 1 \\
\hline Empírica, con técnicas cuantitativas & 28 \\
\hline Análisis de contenido cuantitativo & 18 \\
\hline Análisis secundario de datos y análisis de contenido cuantitativo & 1 \\
\hline Análisis sistemático integrativo & 1 \\
\hline Encuesta & 3 \\
\hline Encuesta online & 4 \\
\hline Investigación experimental & 1 \\
\hline Empírica, con técnicas cuantitativas y cualitativas & 4 \\
\hline Análisis secundario de datos y entrevistas & 1 \\
\hline Encuesta y análisis de contenido cualitativo & 1 \\
\hline Entrevistas en profundidad y análisis de contenido cuantitativo & 1 \\
\hline Análisis de contenido cuantitativo y análisis de contenido cualitativo & 1 \\
\hline Total & 44 \\
\hline
\end{tabular}

Fuente: elaboración propia.

Estos datos demuestran que la línea principal de investigación presente en las revistas analizadas es la de los trabajos empíricos utilizando técnicas cuantitativas, ya que buscan resultados tangibles y fácilmente medibles. La tendencia actual es la de facilitar un método útil a las empresas para que puedan gestionar el engagement 
en las redes sociales, por lo que los trabajos se centran en demostrar la idoneidad o la adaptación de los métodos existentes más que en el estudio de nuevos métodos.

\section{DISCUSIÓN Y CONCLUSIONES}

Tras analizar todos los datos a través de los diversos indicadores, queda patente la importancia que está adquiriendo el engagement en redes sociales para la investigación de comunicación y relaciones públicas. Es especialmente notable el número de artículos referentes al análisis de la responsabilidad social corporativa, la comunicación de crisis, la legitimidad o la reputación, debido al creciente interés que tienen las empresas en mejorar y profundizar en la relación que mantienen con sus públicos tanto internos como externos. El desarrollo de las redes sociales ha orientado, en los últimos años, la investigación hacia Facebook y Twitter, circunstancia que puede cambiar próximamente con la popularidad de otras plataformas.

Cabe destacar asimismo, que en este tema, como en muchos otros, las instituciones universitarias estadounidenses han tomado la iniciativa de la producción científica.

Uno de los aspectos más interesantes en el análisis es la constatación de que a pesar de que el objeto de estudio es novedoso y podría tener mucho sentido explorarlo en profundidad desde perspectivas teóricas o con técnicas empíricas cualitativas, predomina el uso de metodologías cuantitativas. Corroboramos lo observado en estudios previos respecto a que los resultados de algunos indicadores como el número de autores, las autorreferencias o la elección metodológica pueden apuntar a criterios relacionados con la proyección de carrera de los propios investigadores.

Las principales limitaciones de este estudio se pueden reflejar desde dos vertientes: la selección muestral y el uso de indicadores bibliométricos. No obstante la luz de otros bibliométricos ya publicados en el ámbito, se puede estimar que el tamaño muestral es el adecuado para este tipo de análisis y para el objeto de estudio.

Hemos observado que el indicador de colaboración institucional, que es una foto fija de la vinculación contractual de los autores a una institución universitaria en el momento de publicación, puede ofrecer una visión errónea de la heterogeneidad institucional. Teniendo en cuenta la movilidad laboral en Estados Unidos y su preponderancia en la formación de postgrado de académicos de otros países, especialmente países emergentes, es probable que la concentración institucional, a partir de otros parámetros, sea aún mayor que la representada.

Por otra parte, hay numerosos indicadores bibliométricos que no hemos utilizado en este trabajo, como el índice de producción, el índice de transitoriedad o el análisis de citas comunes, e incluso otros tantos que no hemos especificado en este estudio, como el índice de Lotka o el índice h, entre otros, que requerirían un mayor número de codificadores.

El presente trabajo supone una aproximación al concepto de engagement en redes sociales desde la interacción entre la comunicación de marca y las relacio- 
68 nes públicas. Es, además, una primera recopilación de la investigación producida sobre el objeto de estudio en el ámbito de relaciones públicas. Este compendio puede servir para futuros investigadores que quieran profundizar en este objeto desde el punto de vista de la gestión de comunicación no solo por la recopilación de trabajos, sino fundamentalmente por su clasificación temática y su orientación hacia el campo de las relaciones públicas.

\section{Agradecimientos}

Agradecimientos a Ludmila Kirchenca por su inestimable aportación a este equipo de trabajo.

Ángeles Moreno-Fernández (mariaangeles. moreno@urjc.es) es doctora con premio extraordinario por la Universidad Pontificia de Salamanca y profesora titular en la Facultad de Comunicación de la Universidad Rey Juan Carlos (Madrid). Ha enseñado e impartido cursos en diversas instituciones educativas en Europa y América. Es presidenta electa de la European Public Relations Research and Education Association, miembro activo del Grupo de Estudios Avanzados en Comunicación (GEAC) de la URJC y ha participado y dirigido proyectos de investigación de la Unión Europea, la European Public Relations Research and Education Association (EUPRERA), el Plank Center de la Universidad de Alabama, la University of Florida y el

Maria Cristina Fuentes-Lara (cristina.fuentes@urjc.es) es doctora por la Universidad de Granada, Universidad Pablo de Olavide y Universidad de Jaén. Actualmente es profesora en la Universidad Rey Juan Carlos e investigadora de la Universidad Francisco de Vitoria. Además de contar con experiencia docente en cursos y seminarios en universidades españolas, marroquíes y latinoamericanas. Es miembro fundador del Observatorio de la Realidad
MICINN. Forma parte desde hace doce años del equipo de investigación del mayor estudio sobre la gestión de comunicación en el mundo, el European Communication Monitor y es directora del proyecto Latin American Communication Monitor. Sus trabajos han sido premiados internacionalmente por la International Communication Association, la Public Relations Society of America y la European Public Relations and Research Association. Ha publicado trece libros, diversos capítulos y más de cincuenta artículos en las revistas internacionales académicas más prestigiosas de su campo. Así mismo, es revisora de diversos comités científicos para revistas nacionales e internacionales, asociaciones e instituciones públicas.

Sociofronteriza del Mediterráneo (OARS del MED) de la Universidad de Granada; miembro del Grupo de Estudios Avanzados en Comunicación (GEAC) de la Universidad Rey Juan Carlos y miembro de la European Public Relations Research and Education Association (EUPRERA). Sus líneas de investigación son género, comunicación y migraciones, fruto de las cuales recibió el Premio Nacional a la Lucha contra la Violencia de Género en 2018. 


\section{Bibliografía}

Bowden, J. L. H. (2009). "The Process of Customer Engagement: A Conceptual Framework". Journal of Marketing Theory and Practice, 17 (1), pp. 63-74.

Brodie, R.; Juric, B.; Ilic, A. y Hollebeek, L. (2011). "Customer Engagement: Conceptual Domain, Fundamental Propositions, and Implications for Research". Journal of Service Research, 14(3), pp. 252-271.

Castillo, A. y Carretón, M. C. (2010). "Investigación en Comunicación. Estudio Bibliométrico de las Revistas de Comunicación en España". Comunicación y Sociedad, 23(2), pp. 289-327.

Dessart, L.; Veloutsou, C. y Morgan-Thomas, A. (2015). "Consumer Engagement in Online Brand Communities: A Social Media Perspective". Journal of Product \& Brand Management, 24, pp. 28-42.

Dhanesh, G. S. (2017). "Putting Engagement in Its PRoper Place: State of the Field, Definition and Model of Engagement in Public Relations". Public Relations Review, 43(5), pp. 925-933.

Di Gangi, P. M. y Wasko, M. M. (2016). “Social Media Engagement Theory: Exploring the Influence of User Engagement on Social Media Usage". Journal of Organizational and End User Computing (JOEUC), 28(2), pp.53-73.

Doorn, J. van; Lemon, K. N.; Mittal, V.; Nass, S.; Pick, D.; Pirner, P. y Verhoef, P. C. (2010). "Customer Engagement Behavior: Theoretical Foundations and Research Directions". Journal of Service Research, 13(3), pp. 253-266.

Gambetti, R. C. y Graffigna, G. (2010). "The Concept of Engagement: A Systematic Analysis of the Ongoing Marketing Debate". International Journal of Market Research, 52(6), pp. 801-826.

Hollebeek, L. D. (2011). "Demystifying Customer Brand Engagement: Exploring the Loyalty Nexus". Journal of Marketing Management, 27(7-8), pp. 785-807.
Hollebeek, L. D.; Conduit, J. y Brodie, R. J. (2016). "Strategic Drivers, Anticipated and Unanticipated Autcomes of Customer Engagement". Journal of Marketing Management, 32(56), pp. 393-398.

Hollebeek, L. D.; Leventhal, R. y Chen, T. (2014). "Exploring Positively 'versus' Negatively Valenced Brand Engagement: A Conceptual Model". Journal of Product \& Brand Management, 23, pp. 62-74.

IAB (2017). Estudio Anual de Redes Sociales. Obtenido el 31 de diciembre de 2017 en $<$ https://iabspain.es/wp-content/uploads/iab_ estudioredessociales_2017_vreducida.pdf>.

Islam, J. y Rahman, Z. (2016). "The Transpiring Journey of Customer Engagement Research in Marketing: A Systematic Review of the Past Decade". Management Decision, 54(8), pp. 2.008-2.034

Kam Fung; So, K.; King, C.; Sparks, B. y Wang, Y. (2016). "Enhancing Customer Relationships with Retail Service Brands: The Role of Customer Engagement". Journal of Service Management, 27(2), pp. 170-193.

Kietzmann, J. H.; Hermkens, K.; Mccarthy, I. P. y Silvestre, B. S. (2011). "Social Media? Get Serious! Understanding the Functional Building Blocks of Social Media". Business Horizons, 54, pp. 241-251.

Kumar, V.; Donkers, B.; Venkatesan, R.; Wiesel, T. y Tillmanns, S. (2010). "Undervalued or Overvalued Customers: Capturing Total Customer Engagement Value". Journal of Service Research, 13, pp. 297.

Mollen, A. y Wilson, H. (2010). "Engagement, Telepresence and Interactivity in Online Consumer Experience2. Journal of Business Research, 63(9-10), pp. 919-925.

Mpinganjira, M. (2016). "Influencing Consumer Engagement in Online Customer Communities: The Role of Interactivity". Acta Commercii - Independent Research Journal in the Management Sciences, 16(1), pp. 1-10. 
Muñoz-Expósito, M.; Oviedo-García, M. A. y Castellanos-Verdugo, M. (2017). "How To Measure Engagement in Twitter: Advancing a Metric". Internet Research, 27(5), pp. 1.122-1.148.

ONTSI (2017). Estudio de uso y actitudes de consumo de contenidos digitales. Obtenido el 31 de diciembre de 2017 en: <https://www.ontsi.red.es/ontsi/es/content/estudio-de-uso-yactitudes-de-consumo-de-contenidos-digitales>.

Peña, L. J. (2012). “Análisis bibliométrico sobre la producción científica archivística en la Red de Revistas Científicas de América Latina y el Caribe (Redalyc) durante el período 2001-2011". Biblios, 48.

Pritchard, A. (1969). "Statistical Bibliography or Bibliometrics". Journal of Documentation, 25(4), pp. 348-349.

Sancho, R. (1990). "Indicadores bibliométricos utilizados en la evaluación de la ciencia y la tecnología. Revisión bibliográfica". Revista española de documentación científica, 13, pp. 3-4.

Shahbaznejad, H.; Dolan, R. y Tripathi, A. (2017). "The Power of Facebook and Instagram Fans: An Exploration of Fan Comments and Their Effect on Social Media Content Strategy. WeB 2017: Workshop on e-Business 2017". Obtenido de <https://ssrn.com/abstract $=3054984>$.
Sixto, J.; Aguado, N. y Riveiro, R. (2017). "Presencia 2.0 de las pymes gallegas: niveles de participación y 'engagement' con los usuarios". Revista Latina de Comunicación Social, 72, pp. 77-68.

Tague-Sutcliffe, J. (1994). "Quantitative Methods in Documentation". En: Vickery, B. (ed.), Fifty Years of Information Progress: A Journal of Documentation Review, pp. 147-188. Londres: Aslib.

Taylor, M. y Kent, M. L. (2014). “Dialogic Engagement: Clarifying Foundational Concepts". Journal of Public Relations Research, 26(5), pp. 384-398.

Tiago, M. P. y Verrísimo, J. C. (2014). "Digital Marketing and Social Media: Whay bother?". Business Horizons, 57, pp. 703-708.

Vivek, S. D.; Beatty, S. E.; Dalela, V. y Morgan, R. M. (2014). “A Generalized Multidimensional Scale for Measuring Customer Engagement". Journal of Marketing Theory and Practice, 22(4), pp. 401-420.

Zhang, M.; Guo, L.; Hu, M. y Liu, W. (2017). "Influence of Customer Engagement with Company Social Networks on Stickiness: Mediating Effect of Customer Value Creation". International Journal of Information Management, 37(3), pp. 229-240.

\section{ANEXO I Productividad por universidad o institución}

\begin{tabular}{c|l|c|c}
$\mathbf{n}^{\mathbf{0}}$ & Universidad o institución de los autores & total & $\%$ \\
\hline 1 & Universidad de la Mancomunidad de Virginia & 8 & $7,34 \%$ \\
\hline 2 & Universidad de Florida & 7 & $6,42 \%$ \\
\hline 3 & Universidad de Miami & 6 & $5,50 \%$ \\
\hline 4 & Universidad Purdue & 4 & $3,67 \%$ \\
\hline 5 & Universidad de St Thomas & 3 & $2,75 \%$ \\
\hline 6 & Universidad Metodista del Sur & 3 & $2,75 \%$ \\
\hline 7 & Universidad de Economía de Izmir & 3 & $2,75 \%$ \\
\hline
\end{tabular}




\begin{tabular}{|c|c|c|c|}
\hline $\mathrm{n}^{\circ}$ & Universidad o institución de los autores & total & $\%$ \\
\hline 8 & Universidad de Alabama & 3 & $2,75 \%$ \\
\hline 9 & Universidad de San Francisco & 3 & $2,75 \%$ \\
\hline 10 & Universidad IULM & 2 & $1,83 \%$ \\
\hline 11 & Universidad de Waikato & 2 & $1,83 \%$ \\
\hline 12 & Universidad de Siracusa & 2 & $1,83 \%$ \\
\hline 13 & Universidad de Ámsterdam & 2 & $1,83 \%$ \\
\hline 14 & Universidad Estatal de Carolina del Norte & 2 & $1,83 \%$ \\
\hline 15 & Universidad de Búfalo & 2 & $1,83 \%$ \\
\hline 16 & Universidad de Purdue & 2 & $1,83 \%$ \\
\hline 17 & Universidad de Georgia & 2 & $1,83 \%$ \\
\hline 18 & Marist College & 2 & $1,83 \%$ \\
\hline 19 & Universidad de Indiana Bloomington & 2 & $1,83 \%$ \\
\hline 20 & Universidad del Golfo de Ciencia y Tecnología & 2 & $1,83 \%$ \\
\hline 21 & Universidad de Jyväskylä & 2 & $1,83 \%$ \\
\hline 22 & Universidad Estatal de Montclair & 2 & $1,83 \%$ \\
\hline 23 & Universidad de Missouri-Kansas City & 2 & $1,83 \%$ \\
\hline 24 & Universidad Tecnológica de Auckland & 2 & $1,83 \%$ \\
\hline 25 & Universidad de Oklahoma & 2 & $1,83 \%$ \\
\hline 26 & Universidad de Tennessee & 2 & $1,83 \%$ \\
\hline 27 & Universidad Rey Juan Carlos & 1 & $0,92 \%$ \\
\hline 28 & Universidad Estatal de Nueva York & 1 & $0,92 \%$ \\
\hline 29 & Macromedia University & 1 & $0,92 \%$ \\
\hline 30 & Universidad de Oregon & 1 & $0,92 \%$ \\
\hline 31 & Universidad James Madison & 1 & $0,92 \%$ \\
\hline 32 & Universidad de Auburn & 1 & $0,92 \%$ \\
\hline 33 & IE Business School & 1 & $0,92 \%$ \\
\hline 34 & Universidad de Royal Roads & 1 & $0,92 \%$ \\
\hline 35 & Universidad Estatal de Connecticut Central & 1 & $0,92 \%$ \\
\hline 36 & Colegio de Charleston & 1 & $0,92 \%$ \\
\hline 37 & Universidad Hankuk de Estudios Extranjeros & 1 & $0,92 \%$ \\
\hline 38 & Universidad de Shanghai & 1 & $0,92 \%$ \\
\hline
\end{tabular}




\begin{tabular}{|c|c|c|c|c|}
\hline 72 & no & Universidad o institución de los autores & total & $\%$ \\
\hline \multirow{23}{*}{ 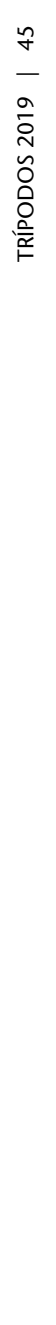 } & 39 & Universidad Bautista de Hong Kong & 1 & $0,92 \%$ \\
\hline & 40 & Universidad de Butler & 1 & $0,92 \%$ \\
\hline & 41 & Edelman Public Relations & 1 & $0,92 \%$ \\
\hline & 42 & Universidad de Dayton & 1 & $0,92 \%$ \\
\hline & 43 & Universidad Estatal de Alabama & 1 & $0,92 \%$ \\
\hline & 44 & Continental Automotive & 1 & $0,92 \%$ \\
\hline & 45 & Universidad Estatal de Cleveland & 1 & $0,92 \%$ \\
\hline & 46 & Universidad de Texas & 1 & $0,92 \%$ \\
\hline & 47 & Universidad de Michigan & 1 & $0,92 \%$ \\
\hline & 48 & La Universidad de Alabama & 1 & $0,92 \%$ \\
\hline & 49 & Universidad Estatal de Oklahoma & 1 & $0,92 \%$ \\
\hline & 50 & Universidad de West Texas A \& M & 1 & $0,92 \%$ \\
\hline & 51 & Universidad de Míchigan Oriental & 1 & $0,92 \%$ \\
\hline & 52 & Universidad de Zagreb & 1 & $0,92 \%$ \\
\hline & 53 & Universidad Massey & 1 & $0,92 \%$ \\
\hline & 54 & Universidad de Maryland & 1 & $0,92 \%$ \\
\hline & 55 & Universidad de Aarhus & 1 & $0,92 \%$ \\
\hline & 56 & Universidad del Sur de Florida & 1 & $0,92 \%$ \\
\hline & 57 & Universidad Srirameshca de Ljubljana & 1 & $0,92 \%$ \\
\hline & 58 & Universidad Dongguk & 1 & $0,92 \%$ \\
\hline & 59 & Universidad Tecnológica de Texas & 1 & $0,92 \%$ \\
\hline & 60 & Universidad en Boston & 1 & $0,92 \%$ \\
\hline & 61 & Universidad en Buffalo & 1 & $0,92 \%$ \\
\hline
\end{tabular}

Fuente: elaboración propia. 